\title{
Modelado espacio-temporal de los servicios que proporciona la biodiversidad en los matorrales de la Cordillera Cantábrica (NO España). Efectos de los cambios socioeconómicos a varias escalas
}

\author{
A. Morán-Ordoñez ${ }^{1, *}$ \\ (1) Departamento de Biodiversidad y Gestión Ambiental, Área de Ecología, Universidad de León, Campus de Vegazana s/n, 24071, León, España. \\ * Autor de correpondencia: A. Morán-Ordóñez [alejandra.moran.ordonez@gmail.com]
}

> Recibido el 20 de agosto de 2013, aceptado el 23 de octubre de 2013

Morán-Ordoñez, A. 2013. Modelado espacio-temporal de los servicios que proporciona la biodiversidad en los matorrales de la Cordillera Cantábrica (NO España). Efectos de los cambios socioeconómicos a varias escalas. Ecosistemas 22(3):124-127. Doi.: 10.7818/ECOS.2013.22-3.17

Los sistemas de montaña son reconocidos a nivel mundial como importantes refugios de diversidad biológica. Sus condiciones ambientales particulares determinadas por marcados gradientes altitudinales, en combinación con las prácticas de uso de baja intensidad a que han sido sometido durante siglos, han favorecido la coexistencia de una amplia diversidad de ecosistemas y especies (MacDonald et al. 2000; Becker y Bugmann 2001). Esta biodiversidad es un elemento clave que determina su capacidad para generar una amplia gama de servicios ecosistémicos de los que se beneficia todo el conjunto de especies que habitan y utilizan esos paisajes, incluyendo las sociedades humanas (MEA 2005). Sin embargo, en el contexto Europeo y desde la segunda mitad del siglo XX, las áreas rurales de montaña han experimentado un marcado proceso de despoblación que ha supuesto el abandono gradual del territorio y la pérdida de las prácticas de manejo tradicional (asociadas al pastoreo extensivo y la agricultura de subsistencia). Estos cambios han derivado en la pérdida de hábitats semi-naturales (aquellos mantenidos por la acción antrópica), favoreciendo en términos generales la homogenización de los patrones paisajísticos y la "renaturalización" del sistema, con sustanciales efectos a corto-medio plazo para la biodiversidad asociada a estos mosaicos heterogéneos, entre otras causas, por la reducción en la diversidad de hábitats disponibles así como por un incremento en el riesgo de incendios asociado a la mayor acumulación de biomasa vegetal (Jongman 2002; Moreira y Russo 2007; Hawksworth y Bull 2008).

En esta Tesis se analizaron los cambios producidos en el paisaje de la vertiente sur de la Cordillera Cantábrica (NO España) (Fig. 1), a diferentes escalas espacio-temporales, identificando las variables ambientales y socio-económicas que han inducido estos cambios. Se dedica especial atención a la puesta en valor de los mosaicos paisajísticos de matorral, pastizal y roca, característicos de los puertos de montaña con pastoreo trashumante.

Este enfoque ha permitido mejorar el conocimiento de los servicios ecosistémicos que estos sistemas proveen a diferentes escalas institucionales (de local a internacional) y definir las bases ecológicas para su gestión.

\section{Cambios en el paisaje: factores inductores de cambio}

El análisis de las dinámicas de cambio del paisaje durante el periodo 1991-2004 permitió evaluar la efectividad de las políticas de conservación implementadas en esta zona, en la que la mayor parte de los espacios protegidos fueron declarados a principio de la década de los 90. A partir de una serie temporal de mapas de ocupación del suelo derivados de la clasificación supervisada de imágenes de satélite LANDSAT, se analizaron las principales dinámicas de cambio en el territorio y se construyeron modelos predictivos espacialmente explícitos con el objeto de determinar, por un lado qué zonas del territorio presentan una mayor probabilidad de cambio, y por otro lado, los factores tanto ambientales como antrópicos que podrían explicar dichos cambios. Los principales cambios observados fueron: (1) expansión del bosque (+ $3 \%)$, que se produjo de forma más marcada en zonas de fondo de valle y el tramo

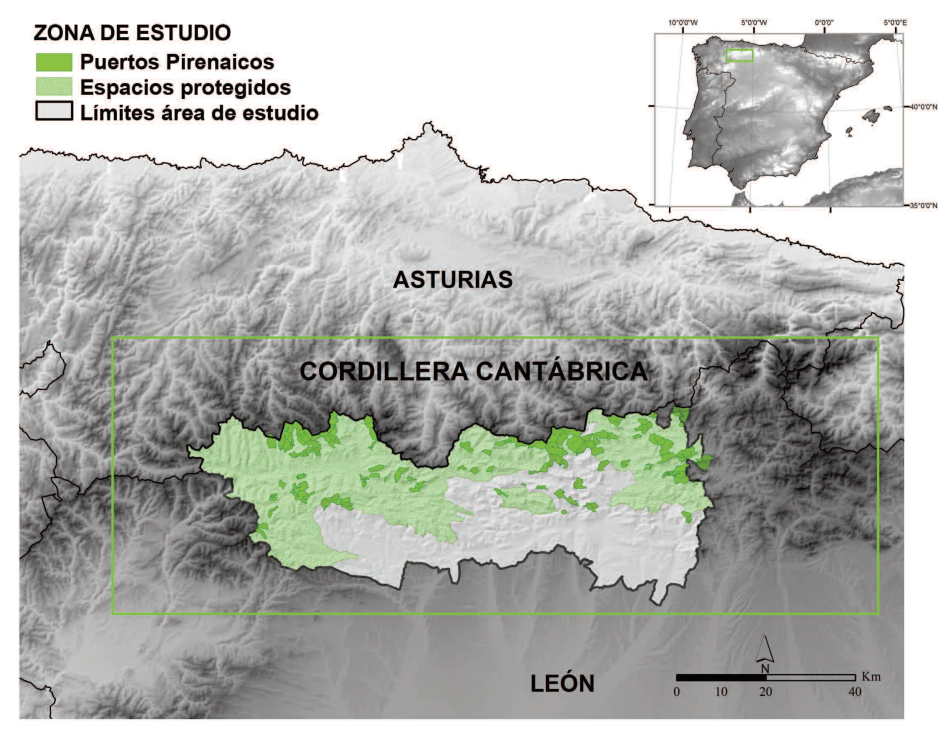

Figura 1. Localización del área de estudio. 
inferior de las laderas próximas a éstos, donde el abandono de la agricultura y las prácticas de manejo tradicional han favorecido su recuperación asociada a procesos de sucesión secundaria; (2) pérdida de las comunidades de matorral (-6\%) ligada a prácticas de gestión (desbroces) fuertemente influenciadas por la demanda de pastos para ganadería de ovino y vacuno, (3) un marcado incremento de áreas de suelo descubierto (+17\%) como consecuencia directa de los desbroces y de la apertura de dos grandes minas a cielo abierto en la segunda mitad de la década de los 90 .

Los patrones generales de estas dinámicas de cambio en la vertiente sur de la Cordillera Cantabrica son dependientes, en parte, del grado de protección del territorio. Así, las medidas de gestión aplicadas en las zonas protegidas -principalmente los desbroces previamente mencionados, encaminados a reducir el riesgo y la propagación de incendios, así como a incrementar la cantidad de pasto disponible para ganado extensivo-, parecen haber favorecido una disminución en la extensión y conectividad de los ecosistemas de matorral que, por el contrario, se incrementaron en las zonas no protegidas. Sin embargo, el grado de protección del territorio no influyó marcadamente en las tendencias de cambio del bosque, que aumentó tanto en área total como en conectividad, en zonas protegidas y en zonas no protegidas.

\section{Las comunidades de matorral: distribución actual y factores ambientales limitantes}

Una vez establecidas las dinámicas de cambio se determinaron los patrones de distribución actuales de cuatro tipos de formaciones de matorral dominadas por Calluna vulgaris, Erica australis, Cytisus purgans-Genista florida y Genista hispanica subsp. occidentalis. Estas comunidades se caracterizan por presentar diferentes estrategias vitales (especialistas vs generalistas) y constituyen hábitats de elevado interés de conservación a nivel Europeo en la vertiente sur de la Cordillera Cantábrica (Directiva Hábitats 92/43/CEE). En concreto, y en base a un algoritmo de Máxima Entropía (MaxEnt: Phillips et al. 2006; Phillips y Dudik 2008), se construyeron modelos de distribución de hábitat para cada una de estas formaciones de manera independiente, usando como variables predictivas las bandas de las imágenes LANDSAT junto con otras variables de carác- ter ambiental (clima, topografía, litología, etc.). Asimismo, y para el caso concreto de las especies Calluna vulgaris y Erica australis, se valoró si la adición de información sobre interacciones bióticas (competencia), mejoraba las predicciones de los modelos de distribución de las formaciones dominadas por estas especies. En definitiva se evaluó si la variación espacial de los factores que limitan la distribución de cada especie podría determinar su éxito bajo condiciones de competencia.

Los modelos de hábitat basados solamente en variables ambientales predijeron con exactitud la distribución de las formaciones de matorral evaluadas. Sin embargo, las estrategias vitales de la especie dominante de cada formación de matorral condicionan los resultados de los modelos. Así, los modelos de las formaciones dominadas por especies especialistas (Calluna vulgaris y Genista hispanica subsp. occidentalis) mostraron un mayor ajuste que los de las dominadas por especies generalistas (Cytisus purgans junto con Genista florida y Erica australis). En este último caso, las predicciones sobreestimaban su distribución. La inclusión de las bandas de las imágenes LANDSAT incrementó el ajuste de los modelos de hábitat y contribuyó a obtener mejores predicciones espaciales, en comparación con los modelos únicamente basados en predictores ambientales (Fig. 2). Las bandas permiten detectar la señal de reflectancia específica de cada formación de matorral, incorporando al modelo información no disponible de otro modo: ocupación del territorio y contenido en humedad del suelo y de la vegetación. Esta aproximación -uso de bandas LANDSAT como variables predictivasmuestra ventajas frente al uso de índices espectrales y mapas derivados de procesos de clasificación de imágenes satélite (generalmente empleados en modelos de distribución de especies), puesto que permite seleccionar la banda más relevante para cada formación, evitando reducir la información espectral a un único índice, así como la incertidumbre asociada al uso de mapas de ocupación como predictores. En el caso de Calluna vulgaris y Erica australis, los modelos más realistas fueron aquéllos que consideraron conjuntamente variables bióticas, abióticas y bandas LANDSAT como predictores. En general, se ha encontrado que cuando dos especies compiten, la especie dominante actúa como factor limitante para la otra especie, forzando a ésta a ocupar zonas libres de competencia.
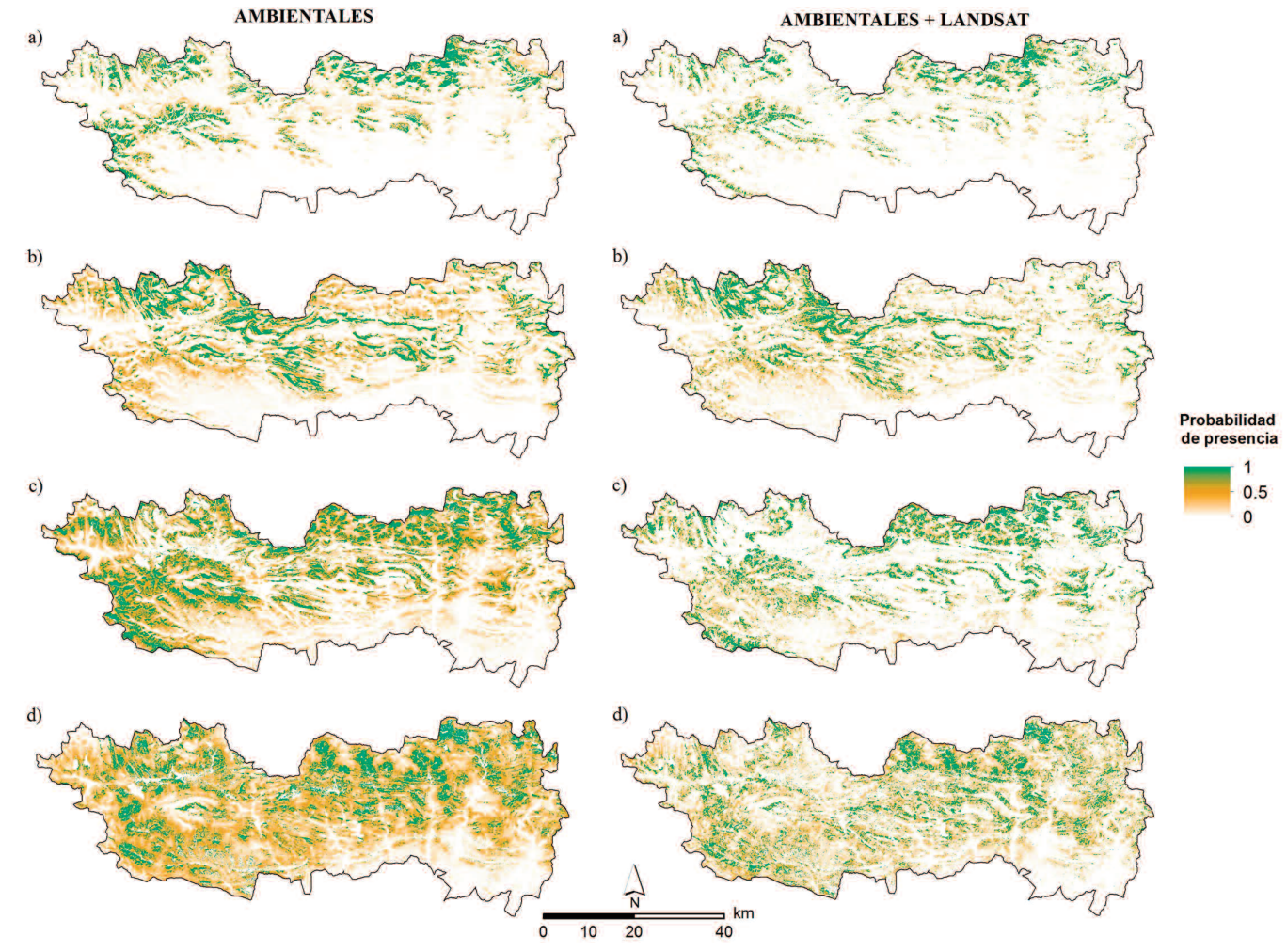

Figura 2. Modelos predictivos de la distribución espacial de las cuatro formaciones de matorral evaluadas en la Cordillera Cantábrica en base a (1) variables ambientales (mapas izquierda) y (2) variables ambientales junto con bandas de las imágenes LANDSAT (mapas derecha): a) Calluna vulgaris; b) Genista hispanica subsp. occidentalis; c) Erica australis; d) Cytisus purgans junto con Genista florida. 


\section{Servicios ecosistémicos asociados a los mosaicos de matorral y pastizal de alta montaña}

Finalmente, se caracterizaron los servicios ecosistémicos proporcionados por los mosaicos paisajísticos de roca, matorral y pastizales montaña de la vertiente sur de la Cordillera Cantábrica (Fig. 3). Estos mosaicos se identifican con los puertos de montaña -o puertos pirenaicos- tradicionalmente aprovechados en época estival por el ganado trashumante. En concreto, se analizó cómo los cambios sociales devenidos desde la segunda mitad del siglo veinte (1950-2010) han modificado tanto los patrones espaciales del paisaje, como la percepción y demanda de los servicios que se pueden obtener de éstos (a distintas escalas institucionales desde local a internacional). Así mismo, se describieron las principales amenazas para la provisión de servicios a diferentes escalas, y se propusieron posibles estrategias de gestión. En los últimos sesenta años la demanda de servicios de aprovisionamiento de dichos mosaicos (a escalas local y regional) ha disminuido significativamente en favor de la demanda de servicios culturales (a escala nacional e internacional) (Tabla 1).

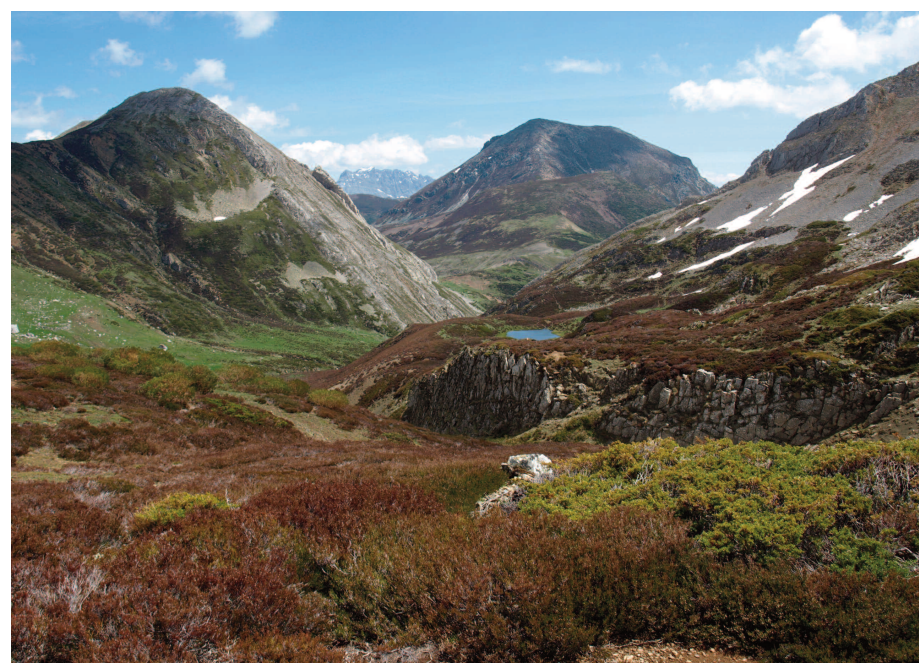

Figura 3. Mosaico paisajístico de matorral, pastizal y roca característico de los puertos de montaña de la montaña Cantábrica.

Tabla 1 Características de los sistemas socio-ecológicos de mediados del siglo XX y actuales asociados a los mosaicos paisajísticos de roca, matorral y pastizal de la Cordillera Cantábrica (puertos de montaña). Para cada sistema, se detallan los servicios de aprovisionamiento, regulación y culturales relacionados con dichos mosaicos paisajísticos, así como las escalas institucionales a las que se demandan éstos servicios: local (L), regional -provincia de León, comunidad Autónoma de Castilla y León- (R), nacional (N) y Europea of Internacional (UE).

\section{SISTEMA SOCIO-ECOLÓGICO}

\section{Mediados siglo XX}

Zonas rurales de la montaña Cantábrica habitadas.

Agricultura de subsistencia en laderas próximas a los pueblos.

Puertos de montaña arrendados a rebaños trashumantes y transterminantes cada año.

El arrendamiento de los puertos de montaña representa una importante fuente de ingresos para los municipios y una fuente de trabajo para los vecinos.

La heterogeneidad de los mosaicos paisajísticos de roca, matorral y pastizales en los puertos de montaña se mantiene mediante el manejo extensivo del ganado y la quema de manchas de matorral para garantizar la disponibilidad de pastos anualmente.

\section{Actualmente}

Abandono rural y envejecimiento de la población de los pueblos.

Desaparición de la economía de subsistencia.

Abandono de los puertos de montaña asociado al marcado declive de las actividades trashumantes y transterminantes de ganado ovino desde la década de los 60 .

El arrendamiento de los puertos de montaña representa un ingreso residual para los ayuntamientos.

Predominio de ganado vacuno y equino en los puertos, que hacen un uso completamente distinto del mosaico paisajístico (mayor consumo de pasto y menor consumo de especies leñosas).

Cambios de usos y prohibición de quemas.

Abandono generalizado del territorio que ha favorecido la expansión del matorral y el bosque. Tendencia hacia la homogeneización del paisaje y la pérdida de biodiversidad.

\section{TIPOS DE SERVICIOS}

ESCALA

\begin{tabular}{ll} 
Aprovisionamiento & \\
\hline Productos como miel, carne, queso,etc. & $\mathrm{L}, \mathrm{R}$ \\
Caza & $\mathrm{L}$ \\
Recursos forrajeros (pastos) & $\mathrm{L}, \mathrm{R}, \mathrm{N}$ \\
Combustible (madera) & $\mathrm{L}$, \\
Fertilizantes & $\mathrm{L}$ \\
Plantas medicinales (e.g. Gentiana lutea) & $\mathrm{L}, \mathrm{N}$
\end{tabular}

\section{Regulación}

\begin{tabular}{ll} 
Prevención erosión suelo & L, R \\
Retención, filtrado y purificación agua & L, R \\
\hline
\end{tabular}

Culturales

Identidad cultural, modo de vida

$\mathrm{L}$

\section{Aprovisionamiento}

Productos como miel, carne, queso, etc.

$L, R$

Recursos forrajeros (pastos)

L, N

Plantas medicinales

$\mathrm{R}, \mathrm{N}, \mathrm{UE}$

Provisión de hábitat para especies de especial interés

$R, N$, UE de conservación

Diversidad genética

N, UE

Regulación

Prevención erosión suelo

$L, R$

Retención y purificación agua

$\mathrm{L}, \mathrm{R}$

Secuestro de carbono y mitigación del cambio

$\mathrm{N}, \mathrm{UE}$ climático

\section{Culturales}

Patrimonio cultural y tradiciones

$\mathrm{R}, \mathrm{N}, \mathrm{UE}$

Caza deportiva

$R, N$, UE

Refugios de biodiversidad

$\mathrm{R}, \mathrm{N}, \mathrm{UE}$

Ecoturismo, importancia por sus valores recreativos 
El sistema socio-ecológico en la década de los años cincuenta dependía principalmente de servicios locales interdependientes. Este sistema se ha transformado en la actualidad en un sistema multi-escalar inestable, en el que la demanda de servicios de estos paisajes por parte de sectores pertenecientes a diferentes escalas institucionales produce situaciones conflictivas en relación su gestión y conservación. En el análisis de un caso práctico de la interacción entre la provisión y demanda de distintos servicios (demanda de pastos para ganadería extensiva y disponibilidad de hábitat adecuado para comunidades de aves) se concluyó que los cambios producidos en los patrones paisajísticos de los puertos de montaña en los últimos sesenta años determinan la actual importancia del servicio de aprovisionamiento de hábitat para las aves nidificantes. Concretamente, aquellas zonas donde el manejo tradicional permite el mantenimiento de mosaicos heterogéneos de pastizal, matorral y roca constituyen zonas de elevada idoneidad para albergar gran riqueza ornítica.

Los resultados de este trabajo han permitido obtener un conocimiento integrado del territorio que puede resultar de gran utilidad para el desarrollo de planes de conservación y gestión en la Cordillera Cantábrica.

\section{Referencias}

Becker, A., Bugmann, H. (Eds.). 2001. Global Change and Mountain Regions- The Mountain Research Initiative. IGBP Secretariat Stockholm, Sweden. 86p.

Hawksworth, D.L., Bull, A.T. (Eds.). 2008. Biodiversity and conservation in Europe. Topics in biodiversity and conservation series (Vol. 7). Springer, The Netherlands. 440p.

Jongman, R.H.G. 2002. Homogenisation and fragmentation of the European landscape: ecological consequences and solutions. Landscape and Urban Planning 58: 211-221.

MacDonald, D., Crabtree, J.R., Wiesinger, G., Dax, T., Stamou, N., Fleury, P., Gutierrez-Lazpita, J., Gibon, A. 2000. Agricultural abandonment in mountain areas of Europe: environmental consequences and policy response. Journal of Environmental Management 59: 47-69.

MEA (Millennium Ecosystem Assessment) 2005. Ecosystems and human well-being: current state and trends. Findings of the Conditions and Trends working group. Island Press, Washington, DC.USA.

Moreira, F., Russo, D. 2007. Modelling the impact of agricultural abandonment and wildfires on vertebrate diversity in Mediterranean Europe. Landscape Ecology 22: 1461-1476.

Phillips, S.J., Anderson, R.P., Schapire, R.E. 2006. Maximum entropy modeling of species geographic distributions. Ecological modelling 190: 231-259.

Phillips, S.J., Dudík, M. 2008. Modeling of species distributions with Maxent: new extensions and a comprehensive evaluation. Ecography 31: 161-175.

\section{ALEJANDRA MORÁN-ORDÓÑEZ}

\section{Modelado espacio-temporal de los servicios que proporciona la biodiversidad en los matorrales de la Cordillera Cantábrica (NO España). Efectos de los cambios socioeconómicos a varias escalas.}

\section{Tesis Doctoral}

Departamento de Biodiversidad y Gestión Ambiental, Universidad de León

Mayo 2012

Directores: Dr. Estanislao de Luis Calabuig, Dra. Leonor Calvo y Dra. Susana Suárez-Seoane

\section{Publicaciones resultantes de la tesis}

Morán-Ordóñez, A., Suárez-Seoane, S., Calvo, L., de Luis, E. 2011. Using predictive models as a spatially explicit support tool for managing cultural landscapes. Applied Geography 31: 839-848.

Morán-Ordóñez, A., Suárez-Seoane, S., Elith, J., Calvo, L., de Luis, E. 2012. Satellite surface reflectance improves habitat distribution mapping: a case study on heath and shrub formations in the Cantabrian Mountains (NW Spain). Diversity and Distributions 18: 588-602.
Morán-Ordóñez, A., Bugter, R., Suárez-Seoane, S., de Luis, E., Calvo, L. 2013. Temporal Changes in Socio-Ecological Systems and Their Impact on Ecosystem Services at Different Governance Scales: A Case Study of Heathlands. Ecosystems 16: 765-782.

Morán-Ordóñez, A., Suárez-Seoane, S., Marcos,E., Luis, E. and Calvo, L. 2013. The heathlands Economy in South-West Europe: Cantabrian Mountain (Spain). En: Diemont, W.H., Siepel H., Webb, N.R. (eds), Economy and Ecology of Heathlands, pp 93-104. KNNV Uitgeverij Publishing, The Netherlands. ISBN: 9789050114615. 